\title{
Mismatch between Probiotic Benefits in Trials versus Food Products
}

\author{
Mary J. Scourboutakos ${ }^{1}$, Beatriz Franco-Arellano ${ }^{1}$, Sarah A. Murphy ${ }^{1}$, Sheida Norsen ${ }^{1}$, \\ Elena M. Comelli ${ }^{1,2, *}$ and Mary R. L'Abbé ${ }^{1,2, *}$ \\ 1 Department of Nutritional Sciences, Faculty of Medicine, University of Toronto, Toronto, ON M1E 3S1, \\ Canada; m.scourboutakos@mail.utoronto.ca (M.J.S.); beatriz.francoarellano@mail.utoronto.ca (B.F.-A.); \\ sarah.murphy@mail.utoronto.ca (S.A.M.); sheida.norsen@alum.utoronto.ca (S.N.) \\ 2 Center for Child Nutrition and Health, Faculty of Medicine, University of Toronto, Toronto, \\ ON M1E 3S1, Canada \\ * Correspondence: elena.comelli@utoronto.ca (E.M.C.); mary.labbe@utoronto.ca (M.R.L.); \\ Tel.: +1-416-978-6284 (E.M.C.); +1-416-978-7235 (M.R.L.)
}

Received: 10 February 2017; Accepted: 6 April 2017; Published: 19 April 2017

\begin{abstract}
Probiotic food products contain a variety of different bacterial strains and may offer different health effects. The objective was to document the prevalence and dosage of probiotic strains in the Canadian food supply and to review the literature investigating these strains in order to understand what health benefits these products may offer. The Food Label Information Program was used to identify probiotic-containing products in the food supply. PubMed, Web of Science, and Embase were searched for randomized controlled trials that tested the health effects of these strains in humans. There were six probiotic strains/strain combinations identified in the food supply. Thirty-one studies investigated these strains and found that they are associated with decreased diarrhea and constipation, improved digestive symptoms, glycemic control, antioxidant status, blood lipids, oral health, and infant breastfeeding outcomes, as well as enhanced immunity and support for Helicobacter pylori eradication. There were a limited number of studies investigating these strains. Many studies were funded by the food industry and tested dosages that were up to twenty-five times the dosage found in most food products. Probiotic food products could have health benefits not currently reported on their labels. However, many dosages are too low to provide the benefits demonstrated in clinical trials. Further research is needed to enable more effective use of these functional foods.
\end{abstract}

Keywords: probiotics; yogurt; functional foods; microbiome; dairy products; food supply; packaged foods; Canada; public health; preventive medicine

\section{Introduction}

Probiotics are "live microorganisms that when administered in adequate amounts confer a health benefit on the host" [1,2]. The benefits of consuming bacteria have been known since ancient times, when fermented milk was commonly prescribed to treat an upset stomach [3]. Today, the term "probiotic" has been defined and qualified by the World Health Organization, which put also forward guidelines to support their use. Accordingly, different probiotics have been shown to prevent or treat a wide range of health issues, including respiratory tract infections, infectious diarrhea, atopic eczema associated with cow's milk allergy, infant colic, necrotizing enterocolitis, pouchitis, bacterial vaginosis, Clostridioides (formerly Clostridium) difficile-associated diarrhea, and urinary tract infections [4-6].

Probiotic food products are one of the fastest growing product markets globally [7]. Currently, commercial probiotic food products contain a variety of different probiotic species and strains. Certain health benefits are common to most or all probiotic species. These effects are considered "core benefits" 
and include the regulation of intestinal transit, normalization of perturbed microbiota, turnover of enterocytes, competitive exclusion of pathogens, colonization resistance, and short-chain fatty acid production [2]. Meanwhile, some probiotic effects are found only among specific species of probiotics. Examples include vitamin synthesis, gut-barrier reinforcement, bile salt metabolism, enzymatic activity, and neutralization of carcinogens [2]. Lastly, certain benefits may only be found among specific strains of bacteria; this includes neurological effects, immunological effects, endocrinological effects, and the production of bioactives [2].

Therefore, probiotic food products currently in the marketplace may have the potential to offer a variety of different health benefits, depending on the specific species and strains of bacteria they contain. However, depending on in which country the products are being sold, consumers have varying degrees of information about the health benefit a probiotic product has been designed to provide.

The WHO has recommended that, where scientific evidence exists, strain specific probiotic health claims should be allowed to enable the linkage of a product to a specific health effect [8]. However, in the European Union, there are no approved probiotic health claims [9]. In fact, even the word "probiotic" is considered a health claim and is not permissible on food packages. In the US, products containing probiotics can state that they "support" the body or "maintain" general well-being (for instance, some products state; "help support your immune system" or "helps naturally regulate the digestive tract") [10]. Meanwhile, in Canada, products contain a general health claim (such as "promotes a healthy gut flora") but could provide more specific benefits depending on the species and strain(s) they contain $[8,11,12]$.

To date, the majority of systematic reviews investigating probiotics have focused on the effects of different strains on a single health outcome or the effects of a single strain on different health outcomes. Furthermore, there have been no reviews focused exclusively on probiotics delivered in food formats.

This study had two objectives; first, to document the prevalence and dosage of probiotic species/strains in the Canadian food supply and, second, to review the literature investigating these species/strains in order to understand what health benefits consumers could potentially receive from the probiotic products in the marketplace.

\section{Materials and Methods}

\subsection{Investigation of Probiotic Strains in the Food Supply}

Data was derived from the Food Label Information Program (FLIP), a database of Canadian food package label information derived from major outlets of the three largest grocery chains in Canada (Loblaws, Metro, and Sobeys) and one major western retailer (Safeway) [13]. This database represents $75.4 \%$ of the grocery retail market share in Canada [14] and provides a detailed assessment of the nutrition information found on Canadian packaged food labels. Grocery store shelves were systematically scanned, and data for every food product with a Nutrition Facts table (NFt), including all available national and private label brands, were collected. Data for food products sold at multiple retailers were collected only once. When multiple sizes of a product were available, only one size was collected. However, all flavors and varieties of a product were collected. Information collected for each product included the Universal Product Code, company, brand, price, Nutrition Facts table information (serving size, calories etc.), ingredients, container size, nutrient content claims, disease risk reduction claims, function claims, front of pack symbols, children's marketing, and other claims (e.g., organic, natural, and gluten-free), in addition to the date and location of sampling. The FLIP database is updated every three years. Presently, two collections have been completed (in 2010 and 2013) and have been described in greater detail elsewhere $[13,15]$. The packages were visually inspected, and ingredient lists of the 15,341 unique products collected in 2013 were searched to identify probiotic-containing products. Fermented foods were not considered to be probiotic products unless they were labeled as being probiotic. The species, strain(s), and dosage found in the 92 probiotic-containing products were recorded and tabulated. In July 2016, Loblaws, Metro, and Sobeys were revisited to identify if 
any probiotic strain and dosage information had changed and to investigate if new probiotic products had entered the marketplace. Four new probiotic products were identified and included in this study. Companies that listed species names without strain information were contacted via e-mail to inquire whether strain data could be disclosed. One company provided strain information via e-mail.

\subsection{Review of Randomized Controlled Trials Testing the Probiotic Strains Found in the Canadian Food Supply}

A systematic search of the peer-reviewed literature investigating each strain/strain combination found in the food supply was conducted in 2016 in accordance with the preferred reporting items for systematic reviews and meta-analysis protocols (PRISMA) checklist (with the exception of items related to meta-analyses) [16]. The full detailed protocol for this is available at PROSPERO registry CRD42106042660 [17].

\subsubsection{Eligibility Criteria}

Study Design, Treatment, and Participants

Double-blind randomized-controlled trials that tested the effects of probiotic strains in the food supply were considered. The probiotic strains were required to be administered in a food format similar to the formats found in the food supply.

Studies that administered probiotics in supplement form, that tested synbiotics, or investigated the safety, tolerance, persistence, or viability of probiotics were not included.

Studies on humans of all ages were considered, with the exception of infants under six-months. Individuals with a chronic disease (like diabetes), infections (such as Helicobacter pylori), or conditions (like constipation or Irritable Bowel Syndrome) were included.

\section{Outcome Measures}

This was not a traditional systematic review. This was an exploratory review and descriptive synthesis that aimed to understand what health effects these food products may offer. Therefore, any and all health-related outcome measures in humans were recorded. This ranged from serum lipid and glycemic levels, to incidence/duration of infections and illness, to markers of inflammation. Effects detected in-vitro were not included. Effects on cellular immunomodulation (e.g., increased number of lymphocytes) were not included.

\subsubsection{Literature Search}

PubMed, Web of Science, and Embase were searched by two independent reviewers (Beatriz Franco-Arellano and Sarah Murphy) from the earliest record to July 2016. The following keywords were searched in the title/abstract: (multiple iterations of each strain name) and (yogurt OR yoghurt OR milk OR fermented milk OR dairy) with (randomized controlled trial) in any field. When the strain was not found in a dairy product, the dairy keywords were omitted. The search was limited to full-manuscripts in English. Only randomized controlled trials in humans were searched.

\section{Study Selection}

After the removal of duplicates, two independent reviewers (Beatriz Franco-Arellano and Sarah Murphy) screened the title and abstracts of retrieved studies against the a priori selection criteria. The selection criteria included any double-blind randomized controlled trial reported in a peer-reviewed journal that included strain/strain combinations found in the food supply, a control group, a quantified dose of the probiotic, a quantified measure of the food treatment, and oral administration of the probiotic via a food format. The study could test any clinical health endpoint on any human population (healthy or sick, including pregnant and breastfeeding mothers). Full-text screening was completed independently by two of the authors of this paper (Mary Scourboutakos and Sarah Murphy), with consensus required for inclusion or exclusion. 


\section{Data Extraction}

The following information was extracted from each manuscript; information related to the article (complete citation plus author, country, and year of publication), the probiotic species and strain(s) tested, strain dosage, food format, population characteristics (e.g., adults, children, male, female, both), health status of the population (e.g., healthy, population with constipation, diabetic population), sample size, study duration, primary outcome measure, secondary outcome measure(s), significant outcomes, and source of funding. Data was independently extracted by one author (Mary Scourboutakos) and verified by a different author (Sarah Murphy). When articles reported insufficient information, attempts were made to contact their authors via-e-mail to retrieve further information.

Assessment of Methodological Quality

The study quality was independently assessed by one author (Mary Scourboutakos) using Health Canada's quality appraisal tool for intervention studies [18] and independently checked by another author (Sarah Murphy). This tool is used to evaluate the quality of studies that provide evidence to support health claim submissions. The risk of bias was assessed using the Cochrane risk-of-bias tool (Table A1) [19].

Data Synthesis

All studies were grouped according to the strain/strain combination they investigated, and health outcomes were recorded accordingly.

\section{Results}

The probiotic strains found in the Canadian food supply and a summary of their health effects are shown in Table 1. The initial search of the probiotic strains found in the food supply and their health benefits yielded 188 papers, with 95 remaining after the removal of duplicates (Figure 1). After reviewing the titles and abstracts, 59 remained for full-text review, 29 of which were eligible for inclusion (Table 2). All studies were deemed to be of a 'high quality' according to Health Canada's quality appraisal tool for intervention studies. The majority of studies were judged to have an overall low risk of bias (Table A1). 


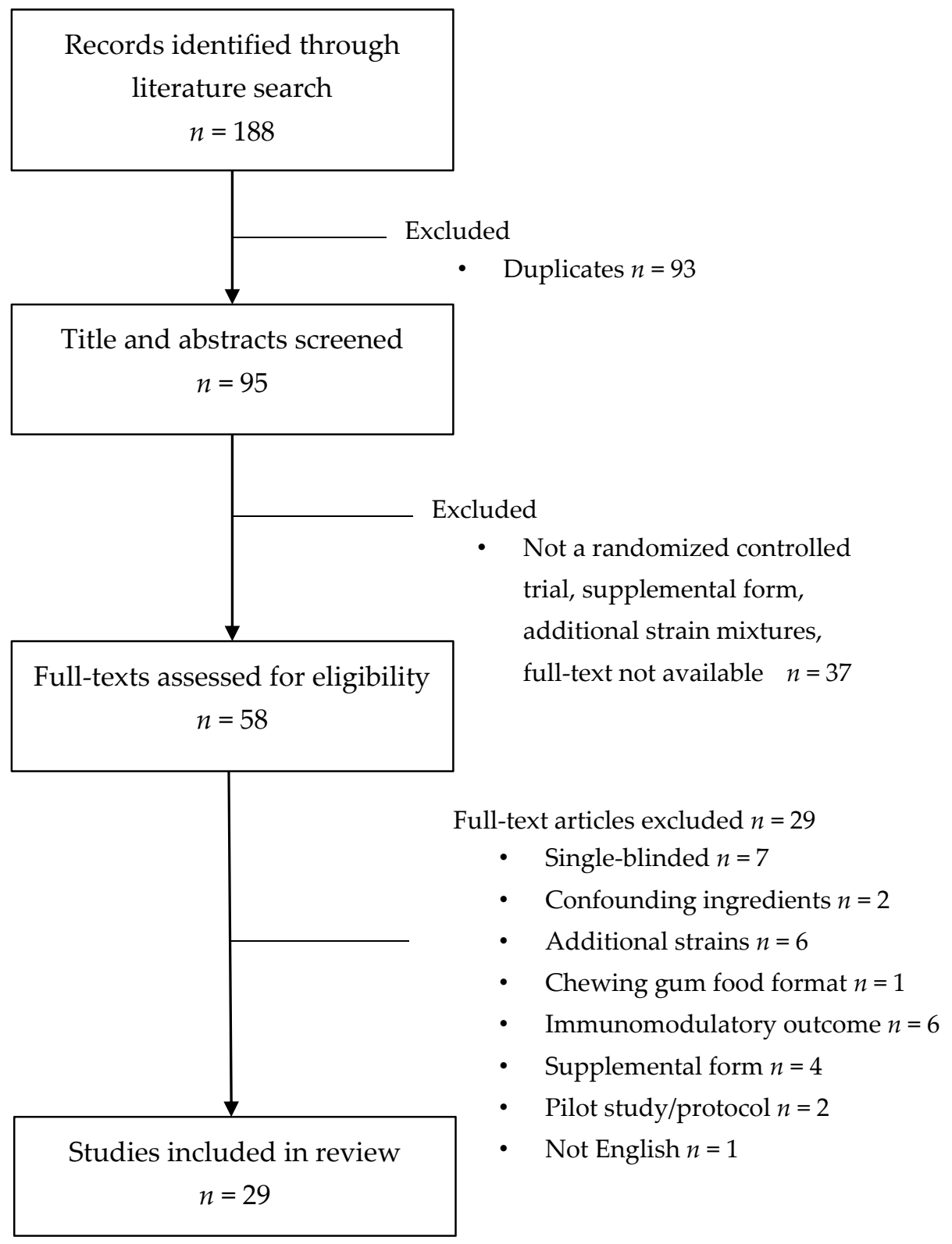

Figure 1. Identification of eligible studies. 
Table 1. Strains in probiotic food products and reported health effects associated with these strains.

\begin{tabular}{|c|c|c|c|c|c|c|c|c|c|c|c|c|c|c|c|c|}
\hline \multirow[b]{2}{*}{ Strain(s) } & \multirow{2}{*}{$\begin{array}{l}\text { Manufacturer } \\
\text { and Product } \\
\text { Brand }\end{array}$} & \multirow[b]{2}{*}{ Food Type } & \multirow{2}{*}{$\begin{array}{c}\text { Probiotic } \\
\text { Dosage in Food } \\
\text { (CFU*/Serving) }\end{array}$} & \multirow{2}{*}{$\begin{array}{l}\text { Dosage Tested in } \\
\text { Studies } \\
\text { (CFU */Day) }\end{array}$} & \multirow[b]{2}{*}{$\begin{array}{c}\text { Duration of } \\
\text { Study }\end{array}$} & \multicolumn{11}{|c|}{ Health Effects Investigated in Healthy Populations } \\
\hline & & & & & & $\begin{array}{c}\text { Acute } \\
\text { Diarrhea }\end{array}$ & $\begin{array}{c}\text { Antibiotic- } \\
\text { Associated } \\
\text { Diarrhea } \\
\end{array}$ & Constipation & $\begin{array}{l}\text { Digestive } \\
\text { Symptoms }\end{array}$ & $\begin{array}{c}\text { Glycemic } \\
\text { Control }\end{array}$ & $\begin{array}{c}\text { Helicobacter } \\
\text { pylor } \\
\text { Eradication }\end{array}$ & Immunity & $\begin{array}{c}\text { Infant } \\
\text { Breastfeeding } \\
\text { Outcomes }\end{array}$ & Inflammation & $\begin{array}{c}\text { Serum } \\
\text { Lipids/Blood } \\
\text { Pressure }\end{array}$ & Oral Health \\
\hline $\begin{array}{c}\text { Bifidobacterium } \\
\text { lactis BB12+ } \\
\text { Lactobacillus } \\
\text { acidophilus } \\
\text { LA-5 } \\
\end{array}$ & $\begin{array}{l}\text { Yoplait's } \\
\text { Yoptimal, } \\
\text { Lucerne's } \\
\text { Organics † } \\
\end{array}$ & Yogurt & $>1 \times 10^{9}$ & $2 \times 10^{6}-3 \times 10^{9}$ & $\begin{array}{l}7 \text { days-6 } \\
\text { weeks }\end{array}$ & & $x[20]^{\$}$ & & & $\mathrm{O}[21]$ & $\mathrm{O}[20]^{\$}$ & & & & $\mathrm{O}[22,23]$ & $x[24,25]$ \\
\hline $\begin{array}{c}\text { Bifidobacterium } \\
\text { lactis BB12 }\end{array}$ & $\begin{array}{l}\text { Iogo's Probio**, } \\
\text { Yoplait's Minigo }\end{array}$ & Yogurt & $>1 \times 10^{9}$ & $1 \times 10^{10}-3.5 \times 10^{10}$ & $\begin{array}{c}10 \text { days }-3 \\
\text { months }\end{array}$ & & & & & & & $\mathrm{o}[26]^{\$}[27]$ & & $\mathrm{O}[28]^{\mathrm{S}}$ & & $\mathrm{x}$ [29] \\
\hline $\begin{array}{c}\text { Lactobacillus } \\
\text { casei } \mathrm{DN} \\
114-001 \\
\end{array}$ & $\begin{array}{l}\text { Danone's } \\
\text { DanActive }\end{array}$ & $\begin{array}{c}\text { Drinkable } \\
\text { yogurt }\end{array}$ & $1 \times 10^{10}$ & $1 \times 10^{10}-3 \times 10^{10}$ & $\begin{array}{c}2 \text { weeks }-6 \\
\text { months }\end{array}$ & $\begin{array}{c}x[30]^{\$,} \\
{[31]^{\circ}} \\
{[32]^{\$}} \\
\end{array}$ & $x[33]^{5}$ & & & & $x[34]^{\$}$ & $\begin{array}{l}X[35,36]^{\$} \\
x[26,30]^{\$}\end{array}$ & $x[37]^{\$}$ & & & \\
\hline $\begin{array}{c}\text { Bifidobacterium } \\
\text { lactis } \\
\text { DN-173 } 010\end{array}$ & $\begin{array}{c}\text { Danone's } \\
\text { Activia }\end{array}$ & Yogurt & $>1 \times 10^{9}$ & $8 \times 10^{9}-2.5 \times 10^{10}$ & $2-4$ weeks & & & $\mathrm{o}[38]^{\mathrm{s}}$ & $\frac{x[39,40]^{\$} x}{[38]^{\$}}$ & & & & & & & $\mathrm{O}[41]^{\mathrm{S}}$ \\
\hline $\begin{array}{c}\text { Lactobacillus } \\
\text { acidophilus } \\
\text { NCFM + } \\
\text { Bifidobacterium } \\
\text { lactis Bi-07 }\end{array}$ & Astro's BioBest & Yogurt & $1 \times 10^{9}$ & $1 \times 10^{10}$ & 6 months & $\mathrm{o}[42]^{\$}$ & & & & & & $x[42]^{s}$ & & & & \\
\hline $\begin{array}{c}\text { Lactobacillus } \\
\text { acidophilus } \\
\text { NFM }\end{array}$ & $\begin{array}{c}\text { President's } \\
\text { Choice's } \\
\text { ProAdvantage } \\
\end{array}$ & Yogurt & $1 \times 10^{9}$ & $1 \times 10^{10}$ & 6 months & $\mathrm{o}[42]^{\mathrm{s}}$ & & & & & & $x[42]^{\mathrm{s}}$ & & & & \\
\hline
\end{tabular}

$\mathrm{X}=$ beneficial effects observed in healthy adults; $\mathrm{x}=$ beneficial effects observed in healthy children, $\mathrm{O}=$ studies that have investigated this outcome and have found no significant effect in adults, $\mathrm{o}=$ studies that have investigated this outcome and found no significant effect in children, $\$=$ indicates that the research was funded by the company that uses that particular strain in their products. A blank square indicates that no research investigating the effects of that strain/strain combination was identified during the systematic review of all literature published up to 21 July 2016, as described in the methods. All effects reported in this table were found in healthy populations that were not diagnosed with a chronic disease or condition. Definition of health effects: Constipation = improved stool frequency, consistency, or condition; Acute diarrhea = decreased incidence or severity of acute diarrhea; Antibiotic-associated diarrhea $=$ decreased incidence of antibiotic-associated or Clostridium difficile-associated diarrhea; Digestive symptoms = decreased abdominal pain/discomfort, bloating, flatulence, or overall GI well-being; Glycemic control = improved fasting glucose, insulin, HbA1c (marker of long-term glycemic control), or HOMA-IR (measure of insulin sensitivity); Helicobacter pylori eradication $=$ enhanced eradication of Helicobacter pylori infections; Immunity = decreased incidence and /or duration of common infectious diseases, including fever, cough, common respiratory infections (rhinitis, sore throat), common gastrointestinal infections (gastroenteritis, vomiting), asthma, or days missed from school; Infant breastfeeding outcomes = infants (2-6 months old) of mothers who consume this strain while breastfeeding had decreased incidence of gastrointestinal episodes and lower medication-use rates; Inflammation = decreased levels of inflammatory markers (ex. C-reactive protein); Lipids = decreased serum total cholesterol, low density lipoprotein (LDL), triglyceride levels, or increased high density lipoprotein (HDL); Oral health = decreased levels of cavity causing bacteria. * CFU = colony forming units. ** Iogo's Probio reported two strains on its label in 2013 (Bifidobacterium lactis BB12 + Lactobacillus acidophilus LA-5) and only one strain on its label in 2016. + These products were available in 2013 but may no longer be available in the Canadian market. Note: All cited references were deemed to be of high quality according to Health Canada's quality appraisal tool for intervention studies [18] 
Table 2. Results of the review of randomized controlled trials investigating the health effects of probiotic strains found in the Canadian food supply ${ }^{1}$.

\begin{tabular}{|c|c|c|c|c|c|c|c|}
\hline Strain & Study, Country (Year) & Population $(n)$ & $\begin{array}{l}\text { Probiotic Dosage } \\
\text { (CFU per Day) }\end{array}$ & Study Duration & Outcome Measures (Primary and Secondary) & $\begin{array}{l}\text { Statistically Significant Effects } \\
\text { (Relative to Placebo Group) }\end{array}$ & Funding Source \\
\hline \multirow{11}{*}{$\begin{array}{l}\text { B. lactis BB12+L. } \\
\text { acidophilus LA-5 }\end{array}$} & $\begin{array}{l}\text { Ivey et al. [21] Australia } \\
\text { (2014) }\end{array}$ & $\begin{array}{c}\text { Overweight adults } \\
n=156\end{array}$ & $3 \times 10^{9}$ & 6 weeks & $\begin{array}{l}\text { Primary: Glycemic control (fasting blood glucose, insulin, } \\
\text { HbA1c, and HOMA-IR) }\end{array}$ & $\begin{array}{l}\text { Increased HOMA-IR (worsened insulin } \\
\text { sensitivity) }\end{array}$ & $\begin{array}{l}\text { Sir Charles Gairdner } \\
\text { Hospital }\end{array}$ \\
\hline & $\begin{array}{l}\text { Sadrzadeh-Yeganeh et al. } \\
\text { [23] Iran (2010) }\end{array}$ & Females $n=90$ & $3.9 \times 10^{7}$ & 6 weeks & $\begin{array}{l}\text { Primary: Serum total cholesterol, HDL, LDL, } \\
\text { and triglycerides }\end{array}$ & No observed effects & Tehran University Grant \\
\hline & $\begin{array}{l}\text { Ivey et al. [22] Australia } \\
\text { (2015) }\end{array}$ & $\begin{array}{c}\text { Overweight adults } \\
n=156\end{array}$ & $3 \times 10^{9}$ & 6 weeks & $\begin{array}{l}\text { Primary: Blood pressure, total cholesterol, HDL, LDL, } \\
\text { and triglycerides }\end{array}$ & No observed effects & $\begin{array}{l}\text { Sir Charles Gairdner } \\
\text { Hospital }\end{array}$ \\
\hline & $\begin{array}{l}\text { deVrese et al. [20] } \\
\text { Germany (2011) }\end{array}$ & $\begin{array}{l}\text { H pylori infected } \\
\text { adults } n=88\end{array}$ & $5 \times 10^{6}$ & 5 weeks & $\begin{array}{l}\text { Primary: Helicobacter pylori activity; Secondary: } \\
\text { Frequency, intensity and duration of abdominal pain; } \\
\text { stool frequency/consistency; duration of diarrhea } \\
\text { episodes; IBS symptoms; orofecal transit time } \\
\end{array}$ & $\begin{array}{l}\text { Decreased duration of } \\
\text { antibiotic-associated diarrhea episodes }\end{array}$ & $\begin{array}{l}\text { Chr. Hansen GmbH J. \& } \\
\text { Co., KG, NOM AG \$ }\end{array}$ \\
\hline & $\begin{array}{l}\text { Ashwin et al. [24] India } \\
\text { (2015) }\end{array}$ & Children $n=60$ & $2 \times 10^{6}$ & 7 days & $\begin{array}{l}\text { Primary: Salivary levels of streptococcus mutans (a cavity } \\
\text { causing bacteria) }\end{array}$ & Reduced salivary mutans streptococci & Funded by study author \\
\hline & $\begin{array}{l}\text { Singh et al. [25] India } \\
\text { (2011) }\end{array}$ & Children $n=40$ & $5.4 \times 10^{7}$ & 10 days & $\begin{array}{l}\text { Primary: Salivary levels of salivary mutans streptococci } \\
\text { and lactobacilli (cavity causing bacteria) }\end{array}$ & Reduced salivary mutans streptococci & Not disclosed \\
\hline & $\begin{array}{l}\text { Ejtahed et al. [43] Iran } \\
\text { (2011) }\end{array}$ & $\begin{array}{l}\text { Type II Diabetics } \\
\qquad n=64\end{array}$ & $>1 \times 10^{9}$ & 6 weeks & $\begin{array}{l}\text { Primary: Fasting blood glucose, HbA1c, insulin and } \\
\text { antioxidant molecules (superoxide dismutase, glutathion } \\
\text { peroxidase, catalase activity, malondialdehyde } \\
\text { concentration, and total antioxidative status) }\end{array}$ & $\begin{array}{l}\text { Decreased fasting blood glucose and } \\
\text { HbA1c; increased activity of superoxide } \\
\text { dismutase, glutathoine peroxidase, and } \\
\text { total antioxidative status }\end{array}$ & Iran Dairy Industry \$ \\
\hline & $\begin{array}{l}\text { Mohamadshahi et al. [44] } \\
\text { Iran (2014) }\end{array}$ & $\begin{array}{l}\text { Type II Diabetics } \\
\quad n=44\end{array}$ & $>1 \times 10^{9}$ & 8 weeks & $\begin{array}{l}\text { Primary: Serum triglycerides, LDL, HDL, triglycerides, } \\
\text { LDL:HDL }\end{array}$ & Decreased LDL:HDL, increased HDL & $\begin{array}{l}\text { Nutrition Disease } \\
\text { Research Center }\end{array}$ \\
\hline & $\begin{array}{l}\text { Ejtahed et al. [45] Iran } \\
\text { (2012) }\end{array}$ & $\begin{array}{l}\text { Type II Diabetics } \\
n=60\end{array}$ & $6 \times 10^{8}$ & 6 weeks & $\begin{array}{l}\text { Primary: total cholesterol, triglycerides, HDL, LDL, total } \\
\text { cholesterol:HDL, LDL:HDL }\end{array}$ & $\begin{array}{l}\text { Decreased total cholesterol, LDL, } \\
\text { LDL:HDL and total cholesterol:HDL }\end{array}$ & $\begin{array}{l}\text { Grant from Tabriz } \\
\text { University }\end{array}$ \\
\hline & $\begin{array}{l}\text { Nabavi et al. [46] Iran } \\
\text { (2014) }\end{array}$ & $\begin{array}{l}\text { Non-alcoholic } \\
\text { fatty liver disease } \\
\text { patients } n=72\end{array}$ & $>1 \times 10^{9}$ & 8 weeks & $\begin{array}{l}\text { Primary: Blood levels of liver enzymes (alanine } \\
\text { aminotransferase and aspartate aminotransferase); fasting } \\
\text { blood glucose; total cholesterol, triglycerides, LDL, HDL. }\end{array}$ & $\begin{array}{l}\text { Decreased blood levels of liver enzymes, } \\
\text { total cholesterol, triglycerides, and LDL }\end{array}$ & $\begin{array}{l}\text { Nutrition Research Center, } \\
\text { Tabriz University }\end{array}$ \\
\hline & $\begin{array}{l}\text { Tonucci et al. [47] Brazil } \\
\text { (2015) }\end{array}$ & $\begin{array}{l}\text { Type II Diabetics } \\
\qquad n=45\end{array}$ & $2 \times 10^{9}$ & 6 weeks & $\begin{array}{l}\text { Primary: Glycemic control (fasting blood glucose, insulin, } \\
\text { HOMA-IR, fructosamine, HbA1c); lipid profile (total } \\
\text { cholesterol, LDL, VLDL, triglycerides, total } \\
\text { cholesterol:HDL); total antioxidant status and cytokine } \\
\text { concentrations (III- }- \text {, Il-10, TNF- } \alpha \text {, adiponectin, and } \\
\text { resistin); fecal short-chain fatty acids }\end{array}$ & $\begin{array}{l}\text { Decreased fructosamine, LDL, and total } \\
\text { cholesterol; significant change in } \mathrm{HbA1c}\end{array}$ & $\begin{array}{l}\text { Brazilian Agri-Research; } \\
\text { Foundation to Support } \\
\text { the State of Miras Gerais }\end{array}$ \\
\hline \multirow{4}{*}{ B. lactis BB12 } & $\begin{array}{l}\text { Caglar et al. [29] Turkey } \\
\text { (2008) }\end{array}$ & $\begin{array}{c}\text { Healthy young } \\
\text { adults } n=24\end{array}$ & $5 \times 10^{8}$ & 10 days & $\begin{array}{l}\text { Primary: Salivary levels of mutans streptococci and } \\
\text { lactobacilli (cavity causing bacteria) }\end{array}$ & Decreased salivary mutans streptococi & Funded by researchers \\
\hline & $\begin{array}{l}\text { Merenstein et al. [48] USA } \\
\text { (2010) }\end{array}$ & Children $n=182$ & $1 \times 10^{10}$ & 90 days & $\begin{array}{l}\text { Primary: Missed days of school due to illness; Secondary: } \\
\text { Diarrhea, stool consistency, respiratory infection, missed } \\
\text { parental work, doctor visits, illnesses, and overall } \\
\text { parental satisfaction }\end{array}$ & No observed effects & The Gerber Foundation $\$$ \\
\hline & $\begin{array}{l}\text { Merenstein et al. [27] USA } \\
\text { (2011) }\end{array}$ & $\begin{array}{l}\text { Healthy children } \\
\qquad n=172\end{array}$ & $1 \times 10^{10}$ & 90 days & $\begin{array}{l}\text { Primary: Missed days of school due to illness; Secondary: } \\
\text { Diarrhea, stool consistency, respiratory infection, missed } \\
\text { parental work, doctor visits, illnesses }\end{array}$ & No observed effects & USDA \\
\hline & $\begin{array}{l}\text { Kekkonen et al. [28] } \\
\text { Finland (2008) }\end{array}$ & $\begin{array}{l}\text { Healthy adults } \\
\quad n=62\end{array}$ & $3.5 \times 10^{10}$ & 3 weeks & $\begin{array}{l}\text { Primary: Blood levels of inflammatory markers including } \\
\text { C-reactive protein and cytokines (TNF- } \alpha, \text { IL- } 6 \text {, } \\
\text { IFN- }- \text {, IL-10) }\end{array}$ & No observed effects & $\begin{array}{l}\text { Resaerch Council Finland } \\
\text { and Valio }\end{array}$ \\
\hline $\begin{array}{l}\text { L. acidophilus } \\
\text { NCFM + B. lactis } \\
\text { Bi- } 07\end{array}$ & $\begin{array}{l}\text { Leyer et al. [42] China } \\
\text { (2009) }\end{array}$ & $\begin{array}{l}\text { Healthy children } \\
\qquad n=326\end{array}$ & $1 \times 10^{10}$ & 6 months & $\begin{array}{l}\text { Primary: Frequency and duration of fever, cough, } \\
\text { rhinorrhea, vomiting, diarrhea, physicians' visits and } \\
\text { antibiotic prescriptions; Secondary: School absences }\end{array}$ & $\begin{array}{l}\text { Decreased incidence of fever, cough, } \\
\text { rhinorrhea, antibiotic use, and days } \\
\text { missed from school. Reduced symptom } \\
\text { duration. }\end{array}$ & Danisco $\$$ \\
\hline
\end{tabular}


Table 2. Cont.

\begin{tabular}{|c|c|c|c|c|c|c|c|}
\hline Strain & Study, Country (Year) & Population $(n)$ & $\begin{array}{c}\text { Probiotic Dosage } \\
\text { (CFU per Day) }\end{array}$ & Study Duration & Outcome Measures (Primary and Secondary) & $\begin{array}{c}\text { Statistically Significant Effects } \\
\text { (Relative to Placebo Group) }\end{array}$ & Funding Source \\
\hline \multirow{4}{*}{$\begin{array}{l}\text { B. lactis DN-173 } \\
\qquad 010\end{array}$} & Pinto et al. [41] Brazil (2013) & $\begin{array}{l}\text { Healthy adults } n= \\
26\end{array}$ & not reported & 2 weeks & $\begin{array}{l}\text { Primary: Salivary levels of cavity-associated microorganisms (mutans } \\
\text { streptococci, lactobacilli and total microorganisms) in saliva }\end{array}$ & No observed effects & Not Disclosed \\
\hline & $\begin{array}{l}\text { Tabbers et al. [38] } \\
\text { Netherlands and Poland } \\
\text { (2011) }\end{array}$ & $\begin{array}{c}\text { Constipated } \\
\text { children } n=159\end{array}$ & $>8 \times 10^{9}$ & 3 weeks & $\begin{array}{l}\text { Primary: Stool frequency; Secondary: Stool consistency, frequency of } \\
\text { faecal incontinence, pain during defecation, abdominal pain, flatulence }\end{array}$ & Decreased flatulence & Danone $^{\$}$ \\
\hline & $\begin{array}{l}\text { Guyonnet et al. [39] Germany } \\
\text { (2009) }\end{array}$ & $\begin{array}{l}\text { Healthy adult } \\
\text { women } n=192\end{array}$ & $2.5 \times 10^{10}$ & 4 weeks & $\begin{array}{l}\text { Primary: Overall GI well-being (intestinal transit, stool frequency and } \\
\text { consistency, abdominal pain/discomfort, bloating, flatulence, stomach } \\
\text { rumbling); Secondary: Frequency of digestive symptoms including } \\
\text { abbominal pain/discomfort, bloating, flatulence, stomach rumbling; } \\
\text { stool frequency and consistency; health-related quality of life }\end{array}$ & $\begin{array}{l}\text { Improved overall GI well-being; } \\
\text { decreased frequency of flatulence, } \\
\text { stomach rumbling, improved stool } \\
\text { consistency, and health-related quality } \\
\text { of life. }\end{array}$ & Danone $\$$ \\
\hline & $\begin{array}{l}\text { Agrawal et al. [40] United } \\
\text { Kingdom (2008) }\end{array}$ & $\begin{array}{l}\text { Adult females } \\
\text { with IBS } n=34\end{array}$ & $2.5 \times 10^{10}$ & 4 weeks & $\begin{array}{l}\text { Primary: Abdominal distension and bloating; Secondary: Orocaecal } \\
\text { and colonic transit times, incidence and severity of IISS symptoms } \\
\text { (abdominal pain/discomfort, bloating, flatulence); overall IBS } \\
\text { symptom severity; time and consistency of bowel movements; feelings } \\
\text { of incomplete evacuation at time of stool passage }\end{array}$ & $\begin{array}{l}\text { Decreased maximal abdominal } \\
\text { distension, orocaecal and colonic transit } \\
\text { times, overall IBS symptom severity, and } \\
\text { abdominal pain/discomfort. }\end{array}$ & Danone $^{\$}$ \\
\hline \multirow{9}{*}{ L. casei DN 114-001 } & $\begin{array}{l}\text { Guillemard et al. [35] } \\
\text { Germany (2010) }\end{array}$ & $\begin{array}{l}\text { Healthy adult shift } \\
\text { workers } n=1000\end{array}$ & $>2 \times 10^{10}$ & 3 months & $\begin{array}{l}\text { Primary: Cumulative number of common infectious diseases (CID) } \\
\text { (e.g., sore throat, sinusitus, nasal discharge, ear ache, influenza, } \\
\text { pneumonia, cough, GI infection, diarrhea, nausea vomiting) } \\
\text { Secondary: Occurrence of having at least one CID: time to first CID, } \\
\text { severity, duration, cumulated duration; occurrence and duration of } \\
\text { fever, sick days, medication use }\end{array}$ & $\begin{array}{l}\text { Decreased occurrence and time to first } \\
\text { CID; decreased duration of fever; } \\
\text { decreased cumulative number of CIDs } \\
\text { (post-hoc analysis) }\end{array}$ & Danone $^{\$}$ \\
\hline & $\begin{array}{l}\text { Merenstein et al. [26] USA } \\
\text { (2010) }\end{array}$ & $\begin{array}{l}\text { Healthy children } \\
\qquad n=638\end{array}$ & $>2 \times 10^{10}$ & 3 months & $\begin{array}{l}\text { Primary: Change in behaviour due to illness (e.g., missed school, } \\
\text { missed sports activity); incidence of common infectious diseases } \\
\text { (CIDs) Secondary: Absences from daycare or school, missed parental } \\
\text { work, days with diarrhea, vomiting, ttomach pain, constipation, runny } \\
\text { nose, cough, decreasing appetite, fever, rash, medication use }\end{array}$ & Decreased incidence of CID & Danone $\$$ \\
\hline & $\begin{array}{l}\text { Guillemard et al. [36] France } \\
\text { (2009) }\end{array}$ & $\begin{array}{l}\text { Elderly adults } \\
\qquad n=1072\end{array}$ & $>2 \times 10^{10}$ & 3 months & $\begin{array}{l}\text { Primary: Cumulative number of all common infectious diseases (CID) } \\
\text { Secondary: The occurrence of CID (defined as the number of subjects } \\
\text { experiencing at least one CID), duration of CID (cumulative and per } \\
\text { episode), time to first CID, severity of CID, fever associated with CID, } \\
\text { occurrence or duration of medication use }\end{array}$ & $\begin{array}{l}\text { Decreased duration of CID episodes and } \\
\text { cumulative duration of CID }\end{array}$ & Danone $^{\$}$ \\
\hline & $\begin{array}{l}\text { Sykora et al. [34] Czech } \\
\text { Republic (2005) }\end{array}$ & $\begin{array}{l}\text { Children } \mathrm{w} / \mathrm{H} \\
\text { Pylori } n=86\end{array}$ & $1 \times 10^{10}$ & 14 days & Primary: Eradication rate of Helicobacter pylori infection & $\begin{array}{l}\text { Increased Helicobacter pylori } \\
\text { eradication rates }\end{array}$ & $\begin{array}{l}\text { Ministry of Health } \\
\text { and Danones }\end{array}$ \\
\hline & $\begin{array}{l}\text { Ortiz-Andrellucchi et al. [37] } \\
\text { Spain (2008) }\end{array}$ & $\begin{array}{l}\text { Breastfeeding } \\
\text { infants } n=104\end{array}$ & $3 \times 10^{10}$ & 6 weeks & $\begin{array}{l}\text { Primary: Immunomodulatory molecules in breast milk (not included } \\
\text { in this review) Secondary: Infant growth and weight; incidence of } \\
\text { gastrointestinal episodes, respiratory symptoms, medication use, } \\
\text { allergies and dermatitis }\end{array}$ & $\begin{array}{l}\text { Reduced incidence of gastrointestinal } \\
\text { episodes and lower rate of medication } \\
\text { use in infants }\end{array}$ & Danone $\$$ \\
\hline & $\begin{array}{l}\text { Agarwal et al. [31] India } \\
\text { (2002) }\end{array}$ & Children $n=150$ & $2-3 \times 10^{10}$ & 9 months & Primary: Duration of acute diarrhea & Decreased duration of acute diarrhea & Not Disclosed \\
\hline & $\begin{array}{l}\text { Hickson et al. [33] United } \\
\text { Kingdom (2007) }\end{array}$ & $\begin{array}{l}\text { Elderly in-patients } \\
n=137\end{array}$ & $2 \times 10^{10}$ & 2 weeks & $\begin{array}{l}\text { Primary: Incidence of antibiotic-associated diarrhea and Clostridium } \\
\text { difficile associated diarrhea }\end{array}$ & $\begin{array}{l}\text { Decreased incidence of antibiotic- and } \\
\text { Clostridium } \text {-associated diarrhea }\end{array}$ & Danone $^{\$}$ \\
\hline & $\begin{array}{l}\text { Giovannini et al. [30] Italy } \\
\text { (2007) }\end{array}$ & $\begin{array}{c}\begin{array}{c}\text { Children with } \\
\text { asthma/rhinitis } \\
n=187\end{array} \\
\end{array}$ & $1 \times 10^{10}$ & 12 months & $\begin{array}{l}\text { Primary: Episodes and duration of asthma and rhinitis (runny/stuff } \\
\text { nose) Secondary: Episodes and duration of abdominal symtoms, } \\
\text { diarrhea and fever }\end{array}$ & $\begin{array}{l}\text { Decreased asthma and rhinitis episodes, } \\
\text { decreased duration of diarrhea in } \\
\text { children with rhinitis }\end{array}$ & Danone $^{\$}$ \\
\hline & Giralt et al. [49] Spain (2008) & $\begin{array}{l}\text { Gynecological } \\
\text { cancer patients } \\
n=85\end{array}$ & $2.8 \times 10^{10}$ & 6 months & $\begin{array}{l}\text { Primary: Frequency and severity of radiation induced diarrhea } \\
\text { Secondary: Time to the development of diarrhea, stool consistency }\end{array}$ & Improved stool consistency & Danone $^{\$}$ \\
\hline
\end{tabular}

${ }^{1}$ All probiotic strains in the Canadian food supply were recorded and a systematic review of their health effects was conducted. All literature published up to 21 July 2016 was included, as described in the methods. All studies included in the review were deemed to be of a 'high quality' according to Health Canada's quality appraisal tool for intervention studies and thus are considered eligible to substantiate a health claim [18]. ${ }^{\$}$ Indicates that funding was provided by the food industry $\mathrm{HbA} 1 \mathrm{c}=$ hemoglobin A1c, a long-term measure of glycemic control; HOMA-IR = a measure of insulin sensitivity; LDL = low-density lipoprotein; HDL = high-density lipoprotein; VLDL = very low-density lipoprotein; IBS = irritable bowel syndrome; $\mathrm{CID}=$ common infectious diseases. 
Danone's DanActive contained one proprietary strain (Lactobacillus casei DN 114-001). This was one of the most well studied strains in the food supply with eleven studies, all funded by Danone, investigating its effects [35]. Three studies showed decreased incidence [26,35] and duration [36] of common infectious diseases (ranging from upper respiratory tract infections to sore throats and influenza) in adults, children, and seniors. Of these, one study showed decreased duration of acute diarrhea in children [31]. One study of hospitalized elderly adults showed decreased incidence of Clostridium difficile and antibiotic-associated diarrhea [33]. Other effects associated with this strain included decreased asthma and rhinitis episodes [30] and increased Helicobacter pylori eradication rates in children [34]. One study tested the effect of this strain when consumed by breastfeeding mothers and showed that their infants had a reduced incidence of gastrointestinal episodes and a lower rate of medication use [37]. The probiotic dosage administered in these studies was up to three times the dosage found in one serving of this product.

Danone's Activia contained a different proprietary strain, Bifidobacterium lactis DN-173 010. This strain was associated with improved overall GI well-being, including decreased flatulence [38], decreased stomach rumbling, and improved stool consistency [39]. In one study of women with irritable bowel syndrome (IBS), this strain was shown to decrease overall IBS symptom severity and to decrease maximal abdominal bloating [40].

President's Choice's ProAdvantage contained Lactobacillus acidophilus NCFM. One study tested this strain in children and found decreased incidence of fever, cough, rhinorrhea, antibiotic use, symptom duration, and days missed from school [42]. However, the dosage tested in the study (10 billion colony forming units ( $\mathrm{cfu}$ ) per day) was ten times the dosage found in the product ( 1 billion cfu per day). Astro's BioBest contained Lactobacillus acidophilus NCFM in combination with Bifidobacterium lactis Bi-07. This combination was tested in the same study reported above and was found to have the same effects and dosage discrepancy.

Bifidobacterium lactis BB-12 was found in two brands; Iogo's Probio and Yoplait's Minigo (a product intended for children). This strain was investigated in four studies. In one study, testing a dosage that was half of what is found in these products, this strain was associated with decreased levels of a cavity causing bacteria (mutans streptococci) in saliva [29]. Two studies tested the effect of this strain (at a dosage that was ten-times the dosage found in the product) on children's risk of illness and absences from school [27,48]. No effects were seen. One study tested a dosage that was thirty-five times the dosage found in the products containing this strain and showed no effect on inflammatory markers (C-reactive protein and cytokines) [28].

Bifidobacterium lactis BB-12 in combination with Lactobacillus acidophilus LA-5 was found in two brands (Yoplait's Yoptimal and Lucerne's Organics). Eleven studies investigated this strain combination. Three studies tested dosages that were substantially smaller than the dosage found in commercial products. Two of those studies showed reduced salivary levels of cavity causing bacteria (Streptococcus mutans) [24,25], while one showed decreased duration of antibiotic-associated diarrhea in patients infected with Helicobacter pylori [20]. Two studies investigated the impact of these strains on blood lipids and found no effects despite the fact that one study tested a dosage that was lower than found in commercial products and the other tested a dosage that was higher [22,23]. One study investigated the effect of these strains on glycemic control [21]. It tested a dosage that was three-times the dosage found in commercial products and found decreased insulin sensitivity. There were four studies that tested the effect of these strains on type-two diabetics and used dosages that were similar to those found in commercial products (Table 3). These studies showed improved glycemic control [43,47], improved blood lipid levels [44,45,47], and enhanced antioxidant status [43] in diabetics.

Nine brands labeled species names without identifying the strain (Table 4). Therefore, strain-specific health benefits could not be inferred for these products. 
Table 3. Strains in probiotic food products and reported health effects in populations with a diagnosed non-communicable disease/condition.

\begin{tabular}{|c|c|c|c|c|c|c|c|c|c|c|c|}
\hline \multirow[b]{2}{*}{ Population } & \multirow[b]{2}{*}{$\begin{array}{l}\text { Strain/Strain } \\
\text { Combination }\end{array}$} & \multirow{2}{*}{$\begin{array}{l}\text { Manufacturer } \\
\text { and Product } \\
\text { Brand }\end{array}$} & \multirow{2}{*}{$\begin{array}{l}\text { Probiotic } \\
\text { Dosage in } \\
\text { Food (CFU } \\
* / \text { Serving) }\end{array}$} & \multirow{2}{*}{$\begin{array}{l}\text { Dosage Tested } \\
\text { in Studies } \\
\text { (CFU*/Day) }\end{array}$} & \multicolumn{7}{|c|}{ Health Effects Investigated in Populations with a Disease/Condition } \\
\hline & & & & & $\begin{array}{l}\text { Antioxidant } \\
\text { Status }\end{array}$ & $\begin{array}{l}\text { Digestive } \\
\text { Symptoms }\end{array}$ & $\begin{array}{l}\text { Glycemic } \\
\text { Control }\end{array}$ & Inflammation & Liver Damage & $\begin{array}{l}\text { Radiation } \\
\text { Induced } \\
\text { Diarrhea }\end{array}$ & $\begin{array}{l}\text { Serum } \\
\text { Lipids }\end{array}$ \\
\hline $\begin{array}{c}\text { Type II } \\
\text { Diabetics }\end{array}$ & \multirow{2}{*}{$\begin{array}{c}\text { Bifidobacterium } \\
\text { lactis BB12+ } \\
\text { Lactobacillus } \\
\text { acidophilus } \\
\text { LA-5 }\end{array}$} & \multirow{2}{*}{$\begin{array}{c}\text { Yoplait's } \\
\text { Yoptimal, } \\
\text { Lucerne's } \\
\text { Organics † }\end{array}$} & \multirow[b]{2}{*}{$>1 \times 10^{9}$} & $\begin{array}{c}6 \times 10^{8} \rightarrow 1 \times \\
10^{9}\end{array}$ & $\mathrm{X}[43]^{\$} \mathrm{O}[47]$ & & $\mathrm{X}[43]^{\$},[47]$ & $\mathrm{O}[47]$ & & & $\mathrm{X}[44,45,47]$ \\
\hline $\begin{array}{l}\text { Patients with } \\
\text { Non-Alcoholic } \\
\text { Fatty Liver } \\
\text { Disease }\end{array}$ & & & & $>1 \times 10^{9}$ & & & & \multicolumn{2}{|r|}{$\mathrm{X}[46]$} & & $x[46]$ \\
\hline $\begin{array}{l}\text { Females with } \\
\text { Irritable Bowel } \\
\text { Syndrome }\end{array}$ & $\begin{array}{c}\text { Bifidobacterium } \\
\text { lactis DN-173 } \\
010\end{array}$ & $\begin{array}{l}\text { Danone's } \\
\text { Activia }\end{array}$ & $>1 \times 10^{9}$ & $2.5 \times 10^{9}$ & & $\mathrm{X}[40]^{\$}$ & & & & & \\
\hline $\begin{array}{l}\text { Gynecological } \\
\text { Cancer } \\
\text { patients } \\
\text { undergoing } \\
\text { radiation } \\
\text { therapy } \\
\end{array}$ & $\begin{array}{l}\text { Lactobacillus } \\
\text { casei DN } \\
114-001\end{array}$ & $\begin{array}{l}\text { Danone's } \\
\text { DanActive }\end{array}$ & $1 \times 10^{10}$ & $2.8 \times 10^{10}$ & & & & & & $x[49]^{\$}$ & \\
\hline $\begin{array}{l}\mathrm{X}=\text { benefic } \\
\text { A blank sq } \\
\text { 21 July 201 } \\
\text { Antioxidan } \\
\text { decreased } \mathrm{f} \\
\text { Inflammati } \\
\text { aminotrans } \\
\text { cholesterol, } \\
\text { references } \mathrm{v}\end{array}$ & $\begin{array}{l}\text { ial effects obser } \\
\text { uare indicates t } \\
6 \text {, as described } \\
t \text { status }=\text { activit } \\
\text { ecal transit time } \\
\text { on = Increased l } \\
\text { ferase and aspa } \\
\text { LDL, or triglyce } \\
\text { vere deemed to }\end{array}$ & $\begin{array}{l}\text { ed; } \mathrm{O}=\text { studie } \\
\text { lat no research } \\
\mathrm{n} \text { the methods. } \\
\text { of superoxide } \\
\text { reduced IBS sy } \\
\text { vels of anti-infla } \\
\text { tate aminotrans } \\
\text { ide levels; incre } \\
\text { e of high qualit }\end{array}$ & $\begin{array}{l}\text { lave investig } \\
\text { vestigating t } \\
\text { ffects report } \\
\text { smutase, glu } \\
\text { ptom severit } \\
\text { matory marl } \\
\text { rase) [a marl } \\
\text { ed HDL; imp } \\
\text { according to }\end{array}$ & $\begin{array}{l}\text { ted this outcom } \\
\text { e effects of that } \\
\text { in this table w } \\
\text { thoine peroxid } \\
\text { Glycemic contr } \\
\text { rs (cytokines: IL } \\
\text { r of decreased li } \\
\text { oved lipid ratios } \\
\text { ealth Canada's }\end{array}$ & $\begin{array}{l}\text { and have foun } \\
\text { strain/strain co } \\
\text { re observed in } \\
\text { se, and total an } \\
1=\text { decreased fa } \\
6 \text {, IL-10, TNF- } \alpha \text {, } \\
\text { ver damage]; Ra } \\
\text { * CFU = colony } \\
\text { uality appraisal }\end{array}$ & $\begin{array}{l}\text { no significa } \\
\text { bination wa } \\
\text { ppulations th } \\
\text { xidant statu } \\
\text { ing blood gl } \\
\text { diponectin, a } \\
\text { ation Induce } \\
\text { rming units. } \\
\text { ol for interv }\end{array}$ & $\begin{array}{l}\text { effects; }{ }^{\$}=\text { inc } \\
\text { dentified duri } \\
\text { were diagnos } \\
\text { Digestive sym } \\
\text { se, insulin, an } \\
\text { resistin) Liver } \\
\text { Diarrhea = inc } \\
\text { This product } n \\
\text { ion studies [18 }\end{array}$ & $\begin{array}{l}\text { cates that the re } \\
\text { the systemati } \\
\text { with a diseas } \\
\text { toms: decrease } \\
\text { /or HbA1c (lor } \\
\text { lamage = decrea } \\
\text { lence and sever } \\
\text { y no longer be }\end{array}$ & $\begin{array}{l}\text { earch was fund } \\
\text { review of all li } \\
\text { or condition D } \\
\text { abdominal dist } \\
\text {-term measure } \\
\text { eed serum levels } \\
\text { ty; Serum Lipid } \\
\text { vailable in the m }\end{array}$ & $\begin{array}{l}\text { by the da } \\
\text { rature pub } \\
\text { nition of } \mathrm{h} \\
\text { sion/pain } \\
\text { blood gluc } \\
\text { liver enzy } \\
\text { decreased } \\
\text { ketplace N }\end{array}$ & $\begin{array}{l}\text { industry. } \\
\text { ed up to } \\
\text { h effects: } \\
\text { comfort, } \\
\text { control); } \\
\text { (alanine } \\
\text { um total } \\
\text { All cited }\end{array}$ \\
\hline
\end{tabular}


Table 4. Additional probiotic products with undetermined health effects.

\begin{tabular}{|c|c|c|c|}
\hline \multicolumn{4}{|c|}{ A: Products whose specific health effects are undetermined because there is no research on the strain/strain combination in their specific food format } \\
\hline Bacterial strains identified in the food database * & Manufacturer and product brand & Food type & Probiotic dosage (CFU) per serving \\
\hline Lactobacillus acidohpilus Bi-07 & Irresistibles' Life Smart & Frozen fruit and yogurt blend & Not indicated \\
\hline Lactobacillus acidophilus LA-5 & Breuggens Yog Active Cereal & Cereal with yogurt flakes & $1 \times 10^{9}$ \\
\hline Bacillus coagulans GBI-30 6086 & ShaSha Co's Spelt Ginger Snaps & Cookies & Not indicated \\
\hline $\begin{array}{c}\text { Lactobacillus acidophilus ATCC 4356T } \\
\text { Lactobacillus helveticus ATCC } 10797 \\
\text { Lactobacillus helveticus ATCC } 12046 \\
\text { Lactobacillus helveticus ATCC 15009T } \\
\text { Lactobacillus kefir ATCC 35411T } \\
\text { Lactobacillus kefir ATCC 8007 } \\
\text { Lactobacillus brevis ATCC 14869T } \\
\text { Lactobacillus brevis ATCC 13648 } \\
\text { Lactobacillus kefirgranum LMG 15132T } \\
\text { Lactobacillus parakefir LMG 15133T } \\
\text { Lactobacillus kefiranofaciens ATCC 43761T } \\
\text { Leuconostoc mesenteroides ATCC 8293T } \\
\text { Leuconostoc mesenteroides LMG 14531 } \\
\text { Leuconostoc mesenteroides LMG 6909T } \\
\text { Leuconostoc pseudomesenteroides ATCC 12291T } \\
\text { Lactococcus lactis subsp. lactis LMG 6890T } \\
\text { Lactococcus lactis LMG 7931 } \\
\text { Lactococcus lactis subsp. cremoris LMG } 6897\end{array}$ & † Liberte's Kefir (effervescent) & Fermented milk & $4.5 \times 10^{10}$ \\
\hline
\end{tabular}


Table 4. Cont.

\begin{tabular}{|c|c|c|c|}
\hline \multicolumn{4}{|c|}{ B: Products whose specific health effects are undetermined because they only indicate the species and not the specific strain of the bacteria they contain } \\
\hline Bifidobacterium & Lucerne's Eating Right & Yogurt & Not indicated \\
\hline Lactobacillus casei & Liberte's BioOrganic & Yogurt & $>1 \times 10^{9}$ \\
\hline \multirow{2}{*}{ Bifidobacterium lactis + Lactobacillus acidophilus + Lactobacillus casei } & Liberte's Classic & \multirow{2}{*}{ Yogurt } & $>1 \times 10^{9}$ \\
\hline & Riviera's Petit Pot Yogurt ** & & $1 \times 10^{9}$ \\
\hline \multirow{2}{*}{ Bifidobacterium lactis + Lactobacillus acidophilus } & Liberte's Goat Yogurt & Yogurt & $>1 \times 10^{9}$ \\
\hline & Skotidakis' Greek Yogurt ** & Yogurt & not indicated \\
\hline $\begin{array}{c}\text { Lactobacillus casei } \\
\text { Lactobacillus acidophilus } \\
\text { Bifidobacterium lactis } \\
\text { Lactobacillus rhamnosus } \\
\text { Lactococcus lactis subsp. cremoris } \\
\text { Lactococcus lactis subsp. lactis } \\
\text { biovar diacetylactis } \\
\text { Lactobacillus delbrueckii subsp. lactis } \\
\text { Lactobacillus delbruecki subsp. bulgaricus } \\
\text { lauconostoc mesenteroides subsp. cremoris }\end{array}$ & Liberte's Kefir (non-effervescent) & Fermented milk & $>1 \times 10^{9}$ \\
\hline $\begin{array}{c}\text { Bifidobacterium infantis } \\
\text { Bifidobacterium lactis } \\
\text { Lactobacillus acidophillus } \\
\text { Lactobacillus fermentum } \\
\text { Lactobacillus lactis } \\
\text { Lactobacillus paracasei } \\
\text { Lactobacillus rhamonus } \\
\text { Lactococcus lactis subsp. Cremoris } \\
\text { Lactococcus lactis subsp. Lactis } \\
\text { Lactococcus lactis subsp. lactis biovar diacetylactis } \\
\text { Lactobacillus delbrueckii subsp. bulgaricus Leuconostoc mesenteroides } \\
\text { Leuconostoc pseudomensenteroides }\end{array}$ & Iogo's Probio Kefir & Fermented milk & $2 \times 10^{9}$ \\
\hline $\begin{array}{c}\text { Bifidobacterium bifidum }+ \text { Bifidobacterium longum subsp longum + } \\
\text { Bifidobacterium animals subsp. lactis }\end{array}$ & President's Choice's Kefir ** & Fermented milk & $2 \times 10^{9}$ \\
\hline Lactobacillus acidophilus + Bifidobacterium lactis & President's Choice's Greek Probiotic** & Yogurt & $1 \times 10^{9}$ \\
\hline
\end{tabular}

* Probiotic containing foods were identified in the Food Label Information Program (FLIP), a database of Canadian food package label information. FLIP data was collected in 2013 from major outlets of the four largest grocery retail chains in Canada. Probiotic species/strain information was obtained from the ingredients list and package of each probiotic product. Data was re-verified in 2016 to ensure that the species and dosage information had not changed. ${ }^{* *}$ These products were not included in the 2013 database but were identified when grocery chains were revisited in 2016. + The strains associated with Liberte's effervescent Kefir are not listed on the product label. This data was obtained via an inquiry with the company. All companies that listed species names without strains were contacted to inquire whether strain data could be disclosed. 
Most products contained one or two different strains. Kefir (here a fermented milk with added probiotics) products had the largest strain and species diversity, as well as the highest dosage (45 billion colony forming units per serving). However, not all kefir products contained this dosage and diversity.

\section{Discussion}

Probiotic food products in the Canadian marketplace contained bacterial strains that were associated with a wide variety of health benefits ranging from enhanced immunity to improved glycemic control in diabetics, suggesting that probiotic products could potentially offer health benefits that are not advertised on their labels. However, many of the current probiotic dosages in products were lower than the dosages tested in randomized controlled trials.

\subsection{Dosage}

In order to obtain many of the health benefits reported in the randomized controlled trials that were reviewed in this study, consumers would need to eat anywhere from two to twenty-five servings of these products each day. The WHO has recommended that "the suggested serving size (on the product label) must deliver the effective dose of probiotics related to the health claim" [8]. Currently most products contain one billion colony forming units (CFU) of probiotics because that is the minimum required in order to provide core benefits and thus be eligible to display the probiotic health claim "promotes a healthy gut flora" in Canada [12]. Therefore, if strain-specific health claims were implemented (in addition to the existing general probiotic health claim), companies would have greater incentive to provide the higher dosages needed to convey some of the health benefits reported in this review.

\subsection{Strain Diversity}

Most products contained one or two strains. However, research has shown that, in some cases, strain mixtures can be more effective than single strains [50-54], as "different strains (that are) targeted toward different ailments can be blended into one preparation", enabling cultures to complement each other's health effects and produce synergistic benefits [55]. For instance, Bifidobacterium lactis BB-12 (found in the food supply) has been shown to have greater gut-adherence when accompanied by Lactobacillus rhamnosus GG (one of the most well-studied probiotic strains [4], which is mainly available in supplement form) [56]. Furthermore, evidence from Leyer et al.'s investigation of Lactobacillus acidophilus NCFM alone and in combination with Bifidobacterium lactis Bi-07 showed that the combination of strains resulted in a lower risk of fever, coughing, and rhinorrhea when compared to the single strain [42]. It is understandable that our results found that fewer products contained strain mixtures, as, presently, food companies have no incentive to utilize strain synergies. Not to mention that single strain probiotics are more easily patentable than multi-strain probiotics [52]. Therefore, current health claims that are based on a single strain encourage the addition of single strains and could therefore be partially responsible for promoting a potentially suboptimal pharmaceutical-like approach to probiotic foods. That being said, it should be noted that not all strain mixtures are beneficial, as strains can antagonize one another. Therefore, research is needed to verify if mixtures are synergistic or antagonistic [50]. It has been previously noted that there is a lack of research on multi-strain probiotics because such research is more difficult to conduct and thus more expensive [57].

\subsection{Strengths and Limitations}

A strength of this study is the use of FLIP to derive information on all marketed products, which is why we chose to focus on the Canadian market as a model. Obviously, different markets will have different probiotic-containing products, which may come with benefits that overlap or differ from those discussed here. It is important that future research focuses on these other markets to the benefit of both the consumers and the industry. Furthermore, many probiotic benefits could vary depending 
on an individual's lifestyle and baseline microbiome. Therefore, it is expected that the health effects noted in this review may not benefit all consumers equivalently.

Limitations include the fact that studies in this review tested various strains, dosages, and health outcomes. Therefore, at this point in time, there is no consensus on what strain, dose, or product is best. For example, while our review showed that one strain (Bifidobacterium lactis DN-173 010) was associated with decreased digestive symptoms, this was the only strain for which this outcome was assessed. Hence, we cannot conclude that other strains/products would not also have these benefits. Therefore, these results show what is known according to the limited amount of literature that currently exists. Additionally, since much of the current research was funded by the companies that sell probiotic products and therefore dictated which strains were studied, there is a need for further research on a broader range of species/strains that is supported by alternate funding bodies.

Despite the WHO's recommendation that genus, species, and strain should be designated on a product's label [8], nearly half of the brands in this study did not disclose strain information. Thus, the potential health benefits for these products could not be deduced.

Previous research has shown that industry funded nutrition-related research may bias conclusions in favor of the sponsors' products [58]. Most of the studies included in this review were funded by the companies making the products $[26,30,32-37,40,59]$ or were published in journals that are funded by the food industry (Table 1) [45,46]. Many of these studies investigated a large number of outcome measures but did not make statistical adjustments to control for testing multiple hypotheses. Furthermore, in many of these studies, the primary outcome measure was not significant, and, instead, significance was detected in secondary outcomes or through post-hoc analyses. Therefore, while these studies were deemed to be of high quality and were published in peer-reviewed journals, elements of their analysis suggest that their results should be interpreted with caution.

\section{Conclusions}

Probiotic food products sold in Canada could offer a variety of health benefits depending on the strain(s) and dosage they contain. That being said, the probiotic dosages contained in most food products are currently too low to provide the benefits shown in clinical trials. Therefore, with higher dosages, or with trails substantiating the current dosage, there is potential for the strains that are already in food products to provide more benefits to the consumer.

Currently there is only a small volume of literature investigating the health benefits of the probiotic strains used in the Canadian food supply. Thus, additional clinical trials, particularly ones that are not sponsored by the food industry, are needed. Hopefully this work will encourage funding and regulatory agencies to fund more research investigating probiotics. A larger number of well conducted studies and clear evidence-based labeling regulations will ultimately help the consumers to make informed choices and derive substantiated benefits form the products they choose to consume.

Overall, considering the wide range of diseases and health conditions for which probiotics have been shown to have benefits, further research to promote the optimal design of probiotic food products is needed to enable more effective use of these functional foods.

Acknowledgments: The project was supported by the Canadian Institutes of Health Research Vanier Scholarship (MS), the McHenry Endowed Chair Award (ML), the Lawson Family Chair in Microbiome Nutrition Research (EC), the Department of Nutritional Sciences Graduate Student Fellowship (BFA), the Canadian Institutes of Health Research Master's Award (SN), and finally, in part, by the Center for Child Nutrition and Health, Faculty of Medicine, University of Toronto (EC, ML). Thank you to the L'Abbé lab FLIP data collection team who assembled the 2013 FLIP database. Elena M. Comelli has received funds from a probiotic company to support research; however, the company was not involved in this study.

Author Contributions: Scourboutakos and L'Abbe had full access to all of the data in the study and take responsibility for the integrity of the data and the accuracy of the data analysis. Scourboutakos and Comelli conceived and designed the study; Scourboutakos, Norsen, Franco-Arellano, Murphy, and L'Abbé acquired the data; Scourboutakos, Comelli, and L'Abbé analyzed and interpreted the data; Scourboutakos wrote the manuscript; Scourboutakos, Norsen, Franco-Arellano, Murphy, and L'Abbé reviewed the manuscript for important intellectual content; L'Abbé and Comelli obtained funding; L'Abbé and Comelli supervised the study. 
Conflicts of Interest: The authors declare no conflict of interest.

\section{Appendix A}

Table A1. Appraisal of the risk of bias of the included studies using the Cochrane risk-of-bias tool.

\begin{tabular}{|c|c|c|c|c|c|c|c|}
\hline Strain Studied & Study & $\begin{array}{l}\text { Sequence } \\
\text { Generation }\end{array}$ & $\begin{array}{l}\text { Allocation } \\
\text { Concealment }\end{array}$ & Blinding & $\begin{array}{c}\text { Incomplete } \\
\text { Outcome Data }\end{array}$ & $\begin{array}{l}\text { Selective } \\
\text { Reporting }\end{array}$ & Overall \\
\hline \multirow{4}{*}{ Bifidobacterium lactis BB12 } & Caglar et al. [29] & $\mathrm{L}$ & $\mathrm{L}$ & $\mathrm{L}$ & $\mathrm{L}$ & $\mathrm{L}$ & $\mathrm{L}$ \\
\hline & Merenstein et al. [48] & $\mathrm{L}$ & $\mathrm{L}$ & $\mathrm{L}$ & U & $\mathrm{L}$ & $\mathrm{L}$ \\
\hline & Merentstein et al. [27] & $\mathrm{L}$ & $\mathrm{L}$ & $\mathrm{L}$ & $\mathrm{U}$ & $\mathrm{L}$ & $\mathrm{L}$ \\
\hline & Kekkonen et al. [28] & $\mathrm{U}$ & $\mathrm{U}$ & $\mathrm{L}$ & $\mathrm{L}$ & $\mathrm{L}$ & $\mathrm{U}$ \\
\hline \multirow{4}{*}{$\begin{array}{l}\text { Bifidobacterium lactis } \\
\text { DN-173 } 010\end{array}$} & Pinto et al. [41] & $\mathrm{L}$ & $\mathrm{L}$ & $\mathrm{L}$ & $\mathrm{L}$ & $\mathrm{L}$ & $\mathrm{L}$ \\
\hline & Tabbers et al. [38] & $\mathrm{L}$ & $\mathrm{L}$ & L & $\mathrm{L}$ & $\mathrm{L}$ & $\mathrm{L}$ \\
\hline & Guyonnet et al. [39] & $\mathrm{U}$ & $\mathrm{U}$ & $\mathrm{L}$ & $\mathrm{L}$ & $\mathrm{L}$ & $\mathrm{U}$ \\
\hline & Agrawal et al. [40] & $\mathrm{U}$ & $\mathrm{U}$ & $\mathrm{L}$ & $\mathrm{L}$ & $\mathrm{L}$ & $\mathrm{U}$ \\
\hline $\begin{array}{c}\text { Lactobacillus acidophilus } \\
\text { NCFM + Bifidobacterium } \\
\text { lactis Bi-07 }\end{array}$ & Leyer et al. [42] & $\mathrm{L}$ & $\mathrm{U}$ & $\mathrm{L}$ & $\mathrm{L}$ & $\mathrm{L}$ & $\mathrm{L}$ \\
\hline \multirow{11}{*}{$\begin{array}{c}\text { Bifidobacterium lactis BB12 + } \\
\text { Lactobacillus acidophilus } \\
\text { LA-5 }\end{array}$} & Ivey et al. [21] & $\mathrm{L}$ & $\mathrm{L}$ & $\mathrm{L}$ & $\mathrm{L}$ & $\mathrm{L}$ & $\mathrm{L}$ \\
\hline & Ivey et al. [22] & $\mathrm{L}$ & $\mathrm{L}$ & L & $\mathrm{L}$ & $\mathrm{L}$ & $\mathrm{L}$ \\
\hline & Sadrzadeh-Yeganeh et al. [23] & U & U & $\mathrm{L}$ & U & $\mathrm{L}$ & U \\
\hline & de Vrese et al. [20] & $\mathrm{U}$ & $\mathrm{U}$ & $\mathrm{L}$ & $\mathrm{L}$ & $\mathrm{L}$ & $\mathrm{U}$ \\
\hline & Ashwin et al. [24] & $\mathrm{U}$ & $\mathrm{U}$ & $\mathrm{L}$ & $\mathrm{U}$ & $\mathrm{L}$ & $\mathrm{U}$ \\
\hline & Singh et al. [25] & $\mathrm{L}$ & $\mathrm{L}$ & $\mathrm{L}$ & $\mathrm{U}$ & $\mathrm{L}$ & $\mathrm{L}$ \\
\hline & Ejtahed et al. [45] & $\mathrm{L}$ & $\mathrm{L}$ & $\mathrm{L}$ & $\mathrm{L}$ & $\mathrm{H}$ & $\mathrm{L}$ \\
\hline & Mohamadshahi et al. [44] & $\mathrm{L}$ & $\mathrm{L}$ & L & $\mathrm{U}$ & $\mathrm{L}$ & $\mathrm{L}$ \\
\hline & Ejtahed et al. [43] & $\mathrm{L}$ & $\mathrm{L}$ & $\mathrm{L}$ & $\mathrm{L}$ & $\mathrm{H}$ & $\mathrm{L}$ \\
\hline & Nabavi et al. [46] & $\mathrm{L}$ & $\mathrm{L}$ & L & $\mathrm{L}$ & $\mathrm{U}$ & $\mathrm{L}$ \\
\hline & Tonucci et al. [47] & $\mathrm{L}$ & $\mathrm{U}$ & $\mathrm{L}$ & $\mathrm{L}$ & $\mathrm{L}$ & $\mathrm{L}$ \\
\hline \multirow{9}{*}{$\begin{array}{c}\text { Lactobacillus casei DN } \\
114-001\end{array}$} & Guillemard et al. [36] & $\mathrm{L}$ & $\mathrm{U}$ & $\mathrm{L}$ & $\mathrm{L}$ & $\mathrm{L}$ & $\mathrm{L}$ \\
\hline & Merenstein et al. [26] & $\mathrm{L}$ & $\mathrm{L}$ & $\mathrm{L}$ & $\mathrm{L}$ & $\mathrm{U}$ & $\mathrm{L}$ \\
\hline & Guillemard et al. [35] & $\mathrm{L}$ & $\mathrm{U}$ & L & $\mathrm{L}$ & $\mathrm{L}$ & $\mathrm{L}$ \\
\hline & Sykora et al. [34] & $\mathrm{L}$ & $\mathrm{H}$ & $\mathrm{L}$ & $\mathrm{L}$ & $\mathrm{L}$ & $\mathrm{L}$ \\
\hline & Ortiz-Andrellucchi et al. [37] & $\mathrm{U}$ & $\mathrm{L}$ & $\mathrm{L}$ & $\mathrm{L}$ & $\mathrm{U}$ & $\mathrm{U}$ \\
\hline & Agarwal et al. [31] & $\mathrm{L}$ & $\mathrm{U}$ & $\mathrm{L}$ & $\mathrm{L}$ & $\mathrm{L}$ & $\mathrm{L}$ \\
\hline & Hickson et al. [33] & $\mathrm{L}$ & $\mathrm{L}$ & $\mathrm{L}$ & $\mathrm{U}$ & $\mathrm{L}$ & $\mathrm{L}$ \\
\hline & Giovannini et al. [30] & $\mathrm{L}$ & $\mathrm{H}$ & $\mathrm{L}$ & $\mathrm{L}$ & $\mathrm{L}$ & $\mathrm{L}$ \\
\hline & Giralt et al. [49] & $\mathrm{L}$ & $\mathrm{L}$ & $\mathrm{L}$ & $\mathrm{L}$ & $\mathrm{L}$ & $\mathrm{L}$ \\
\hline
\end{tabular}

Note: $\mathrm{H}=$ high risk of bias, $\mathrm{L}=$ low risk of bias, and $\mathrm{U}=$ unclear risk of bias.

\section{References}

1. Joint FAO/WHO Expert Consultation on Evaluation of Health and Nutritional Properties of Probiotics in Food Including Powder Milk with Live Lactic Acid Bacteria Probiotcs in Food-Health and Nutritional Properties and Guidelines for Evaluation. Available online: http:/ / www.fao.org/3/a-a0512e.pdf (accessed on 8 September 2016).

2. Hill, C.; Guarner, F.; Reid, G.; Gibson, G.R.; Merenstein, D.J.; Pot, B.; Morelli, L.; Canani, R.B.; Flint, H.J.; Salminen, S.; et al. Expert consensus document. The international scientific association for probiotics and prebiotics consensus statement on the scope and appropriate use of the term probiotic. Nat. Rev. Gastroenterol. Hepatol. 2014, 11, 506-514. [CrossRef] [PubMed]

3. Butel, M.J. Probiotics, gut microbiota and health. Med. Mal. Infect. 2014, 44, 1-8. [CrossRef] [PubMed]

4. Goldin, B.R.; Gorbach, S.L. Clinical indications for probiotics: An overview. Clin. Infect. Dis. 2008, 46, S96-S100. [CrossRef] [PubMed]

5. Kaur, I.P.; Kuhad, A.; Garg, A.; Chopra, K. Probiotics: Delineation of prophylactic and therapeutic benefits. J. Med. Food 2009, 12, 219-235. [CrossRef] [PubMed]

6. Taibi, A.; Comelli, E.M. Practical approaches to probiotics use. Appl. Physiol. Nutr. Metab. 2014, 39, 980-986. [CrossRef] [PubMed]

7. Grand View Research Probiotics Market Analysis by Application (Probiotic Functional Foods \& Beverages, Probiotic Dietary Supplements, Animal Feed Probiotics), by End-Use (Human Probiotics, Animal Probiotics) and Segment Forecasts to 2020. Available online: http:/ / www.grandviewresearch.com/industry-analysis / probiotics-market (accessed on 11 August 2016).

8. World Health Organization. Guidelines for the Evaluation of Probiotics in Food. Available online: http:/ / www.who.int/foodsafety/fs_management/en/probiotic_guidelines.pdf (accessed on 25 January 2016). 
9. Glanville, J.; King, S.; Guarner, F.; Hill, C.; Sanders, M.E. A review of the systematic review process and its applicability for use in evaluating evidence for health claims on probiotic foods in the european union. Nutr. J. 2015, 14, 16. [CrossRef] [PubMed]

10. Saldanha, L.G. Us food and drug administration regulations governing label claims for food products, including probiotics. Clin. Infect. Dis. 2008, 46, S119-S121; discussion: S144-S151. [CrossRef] [PubMed]

11. Canadian Food Inspection Agency Health Claims-Probiotic Claims. Available online: http:/ / www.inspection.gc.ca/food/labelling/food-labelling-for-industry/health-claims/eng/ 1392834838383/1392834887794?chap=9 (accessed on 25 January 2016).

12. Health Canada Guidance Document-The Use of Probiotic Microorganisms in Food. Available online: http://www.hc-sc.gc.ca/fn-an/alt_formats/hpfb-dgpsa/pdf/legislation/probiotics_guidanceorientation_probiotiques-eng.pdf (accessed on 25 January 2016).

13. Bernstein, J.; Schermel, A.; Mills, C.; L'Abbe, M. Total and free sugar content of canadian prepackaged foods and beverages. Nutrients 2016, 8, 582. [CrossRef] [PubMed]

14. Canadian Grocer. Executive Report, Canadian Grocery Industry 2012-2013; Rogers Publishing Limited: Toronto, ON, Canada, 2012.

15. Schermel, A.; Emrich, T.E.; Arcand, J.; Wong, C.L.; L'Abbe, M.R. Nutrition marketing on processed food packages in canada: 2010 food label information program. Appl. Physiol. Nutr. Metab. 2013, 38, 666-672. [CrossRef] [PubMed]

16. Shamseer, L.; Moher, D.; Clarke, M.; Ghersi, D.; Liberati, A.; Petticrew, M.; Shekelle, P.; Stewart, L.A. Preferred reporting items for systematic review and meta-analysis protocols (prisma-p) 2015: Elaboration and explanation. Br. Med. J. 2015, 349, g7647. [CrossRef] [PubMed]

17. PROSPERO International Prospective Register of Systematic Reviews. Available online: http:/ / www.crd. york.ac.uk/PROSPERO/display_record.asp?ID=CRD42016042660 (accessed on 28 June 2016).

18. Health Canada Guidance Document for Preparing a Submission for Food Health Claims. Available online: http://www.hc-sc.gc.ca/fn-an/legislation/guide-ld/health-claims_guidance-orientation_ allegations-sante-eng.php (accessed on 10 July 2016).

19. Higgins, J.P.; Altman, D.G.; Gotzsche, P.C.; Juni, P.; Moher, D.; Oxman, A.D.; Savovic, J.; Schulz, K.F.; Weeks, L.; Sterne, J.A. The cochrane collaboration's tool for assessing risk of bias in randomised trials. Br. Med. J. 2011, 343, d5928. [CrossRef] [PubMed]

20. De Vrese, M.; Kristen, H.; Rautenberg, P.; Laue, C.; Schrezenmeir, J. Probiotic lactobacilli and bifidobacteria in a fermented milk product with added fruit preparation reduce antibiotic associated diarrhea and helicobacter pylori activity. J. Dairy Res. 2011, 78, 396-403. [CrossRef] [PubMed]

21. Ivey, K.L.; Hodgson, J.M.; Kerr, D.A.; Lewis, J.R.; Thompson, P.L.; Prince, R.L. The effects of probiotic bacteria on glycaemic control in overweight men and women: A randomised controlled trial. Eur. J. Clin. Nutr. 2014, 68, 447-452. [CrossRef] [PubMed]

22. Ivey, K.L.; Hodgson, J.M.; Kerr, D.A.; Thompson, P.L.; Stojceski, B.; Prince, R.L. The effect of yoghurt and its probiotics on blood pressure and serum lipid profile; a randomised controlled trial. Nutr. Metab. Cardiovasc. Dis. NMCD 2015, 25, 46-51. [CrossRef] [PubMed]

23. Sadrzadeh-Yeganeh, H.; Elmadfa, I.; Djazayery, A.; Jalali, M.; Heshmat, R.; Chamary, M. The effects of probiotic and conventional yoghurt on lipid profile in women. Br. J. Nutr. 2010, 103, 1778-1783. [CrossRef] [PubMed]

24. Ashwin, D.; Ke, V.; Taranath, M.; Ramagoni, N.K.; Nara, A.; Sarpangala, M. Effect of probiotic containing ice-cream on salivary mutans streptococci (sms) levels in children of 6-12 years of age: A randomized controlled double blind study with six-months follow up. J. Clin. Diagn. Res. JCDR 2015, 9, ZC06-ZC09. [CrossRef] [PubMed]

25. Singh, R.P.; Damle, S.G.; Chawla, A. Salivary mutans streptococci and lactobacilli modulations in young children on consumption of probiotic ice-cream containing bifidobacterium lactis bb12 and lactobacillus acidophilus la5. Acta Odontol. Scand. 2011, 69, 389-394. [CrossRef] [PubMed]

26. Merenstein, D.; Murphy, M.; Fokar, A.; Hernandez, R.K.; Park, H.; Nsouli, H.; Sanders, M.E.; Davis, B.A.; Niborski, V.; Tondu, F.; et al. Use of a fermented dairy probiotic drink containing lactobacillus casei (DN-114-001) to decrease the rate of illness in kids: The drink study. A patient-oriented, double-blind, cluster-randomized, placebo-controlled, clinical trial. Eur. J. Clin. Nutr. 2010, 64, 669-677. [CrossRef] [PubMed] 
27. Merenstein, D.; Gonzalez, J.; Young, A.G.; Roberts, R.F.; Sanders, M.E.; Petterson, S. Study to investigate the potential of probiotics in children attending school. Eur. J. Clin. Nutr. 2011, 65, 447-453. [CrossRef] [PubMed]

28. Kekkonen, R.A.; Lummela, N.; Karjalainen, H.; Latvala, S.; Tynkkynen, S.; Jarvenpaa, S.; Kautiainen, H.; Julkunen, I.; Vapaatalo, H.; Korpela, R. Probiotic intervention has strain-specific anti-inflammatory effects in healthy adults. World J. Gastroenterol. 2008, 14, 2029-2036. [CrossRef] [PubMed]

29. Caglar, E.; Kuscu, O.O.; Selvi Kuvvetli, S.; Kavaloglu Cildir, S.; Sandalli, N.; Twetman, S. Short-term effect of ice-cream containing bifidobacterium lactis bb-12 on the number of salivary mutans streptococci and lactobacilli. Acta Odontol. Scand. 2008, 66, 154-158. [CrossRef] [PubMed]

30. Giovannini, M.; Agostoni, C.; Riva, E.; Salvini, F.; Ruscitto, A.; Zuccotti, G.V.; Radaelli, G. A randomized prospective double blind controlled trial on effects of long-term consumption of fermented milk containing lactobacillus casei in pre-school children with allergic asthma and/or rhinitis. Pediatr. Res. 2007, 62, $215-220$. [CrossRef] [PubMed]

31. Agarwal, K.N.; Bhasin, S.K. Feasibility studies to control acute diarrhoea in children by feeding fermented milk preparations actimel and indian dahi. Eur. J. Clin. Nutr. 2002, 56, S56-S59. [CrossRef] [PubMed]

32. Pedone, C.A.; Bernabeu, A.O.; Postaire, E.R.; Bouley, C.F.; Reinert, P. The effect of supplementation with milk fermented by lactobacillus casei (strain DN-114-001) on acute diarrhoea in children attending day care centres. Int. J. Clin. Pract. 1999, 53, 179-184. [PubMed]

33. Hickson, M.; D'Souza, A.L.; Muthu, N.; Rogers, T.R.; Want, S.; Rajkumar, C.; Bulpitt, C.J. Use of probiotic lactobacillus preparation to prevent diarrhoea associated with antibiotics: Randomised double blind placebo controlled trial. Br. Med. J. 2007, 335, 80. [CrossRef] [PubMed]

34. Sykora, J.; Valeckova, K.; Amlerova, J.; Siala, K.; Dedek, P.; Watkins, S.; Varvarovska, J.; Stozicky, F.; Pazdiora, P.; Schwarz, J. Effects of a specially designed fermented milk product containing probiotic lactobacillus casei DN-114-001 and the eradication of h. Pylori in children: A prospective randomized double-blind study. J. Clin. Gastroenterol. 2005, 39, 692-698. [CrossRef] [PubMed]

35. Guillemard, E.; Tanguy, J.; Flavigny, A.; de la Motte, S.; Schrezenmeir, J. Effects of consumption of a fermented dairy product containing the probiotic lactobacillus casei DN-114-001 on common respiratory and gastrointestinal infections in shift workers in a randomized controlled trial. J. Am. Coll. Nutr. 2010, 29, 455-468. [CrossRef] [PubMed]

36. Guillemard, E.; Tondu, F.; Lacoin, F.; Schrezenmeir, J. Consumption of a fermented dairy product containing the probiotic lactobacillus casei DN-114001 reduces the duration of respiratory infections in the elderly in a randomised controlled trial. Br. J. Nutr. 2010, 103, 58-68. [CrossRef] [PubMed]

37. Ortiz-Andrellucchi, A.; Sanchez-Villegas, A.; Rodriguez-Gallego, C.; Lemes, A.; Molero, T.; Soria, A.; Pena-Quintana, L.; Santana, M.; Ramirez, O.; Garcia, J.; et al. Immunomodulatory effects of the intake of fermented milk with lactobacillus casei DN-114-001 in lactating mothers and their children. Br. J. Nutr. 2008, 100, 834-845. [CrossRef] [PubMed]

38. Tabbers, M.M.; Chmielewska, A.; Roseboom, M.G.; Crastes, N.; Perrin, C.; Reitsma, J.B.; Norbruis, O.; Szajewska, H.; Benninga, M.A. Fermented milk containing bifidobacterium lactis DN-173 010 in childhood constipation: A randomized, double-blind, controlled trial. Pediatrics 2011, 127, e1392-e1399. [CrossRef] [PubMed]

39. Guyonnet, D.; Schlumberger, A.; Mhamdi, L.; Jakob, S.; Chassany, O. Fermented milk containing bifidobacterium lactis DN-173 010 improves gastrointestinal well-being and digestive symptoms in women reporting minor digestive symptoms: A randomised, double-blind, parallel, controlled study. Br. J. Nutr. 2009, 102, 1654-1662. [CrossRef] [PubMed]

40. Agrawal, A.; Houghton, L.A.; Morris, J.; Reilly, B.; Guyonnet, D.; Goupil Feuillerat, N.; Schlumberger, A.; Jakob, S.; Whorwell, P.J. Clinical trial: The effects of a fermented milk product containing bifidobacterium lactis DN-173 010 on abdominal distension and gastrointestinal transit in irritable bowel syndrome with constipation. Aliment. Pharmacol. Ther. 2009, 29, 104-114. [CrossRef] [PubMed]

41. Pinto, G.S.; Cenci, M.S.; Azevedo, M.S.; Epifanio, M.; Jones, M.H. Effect of yogurt containing bifidobacterium animalis subsp. Lactis DN-173 010 probiotic on dental plaque and saliva in orthodontic patients. Caries Res. 2014, 48, 63-68. [CrossRef] [PubMed]

42. Leyer, G.J.; Li, S.; Mubasher, M.E.; Reifer, C.; Ouwehand, A.C. Probiotic effects on cold and influenza-like symptom incidence and duration in children. Pediatrics 2009, 124, e172-e179. [CrossRef] [PubMed] 
43. Ejtahed, H.S.; Mohtadi-Nia, J.; Homayouni-Rad, A.; Niafar, M.; Asghari-Jafarabadi, M.; Mofid, V. Probiotic yogurt improves antioxidant status in type 2 diabetic patients. Nutrition 2012, 28, 539-543. [CrossRef] [PubMed]

44. Mohamadshahi, M.; Veissi, M.; Haidari, F.; Javid, A.Z.; Mohammadi, F.; Shirbeigi, E. Effects of probiotic yogurt consumption on lipid profile in type 2 diabetic patients: A randomized controlled clinical trial. J. Res. Med. Sci. 2014, 19, 531-536. [PubMed]

45. Ejtahed, H.S.; Mohtadi-Nia, J.; Homayouni-Rad, A.; Niafar, M.; Asghari-Jafarabadi, M.; Mofid, V.; Akbarian-Moghari, A. Effect of probiotic yogurt containing lactobacillus acidophilus and bifidobacterium lactis on lipid profile in individuals with type 2 diabetes mellitus. J. Dairy Sci. 2011, 94, 3288-3294. [CrossRef] [PubMed]

46. Nabavi, S.; Rafraf, M.; Somi, M.H.; Homayouni-Rad, A.; Asghari-Jafarabadi, M. Effects of probiotic yogurt consumption on metabolic factors in individuals with nonalcoholic fatty liver disease. J. Dairy Sci. 2014, 97, 7386-7393. [CrossRef] [PubMed]

47. Tonucci, L.B.; Olbrich Dos Santos, K.M.; Licursi de Oliveira, L.; Rocha Ribeiro, S.M.; Duarte Martino, H.S. Clinical application of probiotics in type 2 diabetes mellitus: A randomized, double-blind, placebo-controlled study. Clin. Nutr. 2017, 36, 85-92. [CrossRef] [PubMed]

48. Merenstein, D.J.; Smith, K.H.; Scriven, M.; Roberts, R.F.; Sanders, M.E.; Petterson, S. The study to investigate the potential benefits of probiotics in yogurt, a patient-oriented, double-blind, cluster-randomised, placebo-controlled, clinical trial. Eur. J. Clin. Nutr. 2010, 64, 685-691. [CrossRef] [PubMed]

49. Giralt, J.; Regadera, J.P.; Verges, R.; Romero, J.; de la Fuente, I.; Biete, A.; Villoria, J.; Cobo, J.M.; Guarner, F. Effects of probiotic lactobacillus casei DN-114-001 in prevention of radiation-induced diarrhea: Results from multicenter, randomized, placebo-controlled nutritional trial. Int. J. Radiat. Oncol. Biol. Phys. 2008, 71, 1213-1219. [CrossRef] [PubMed]

50. Chapman, C.M.; Gibson, G.R.; Rowland, I. Health benefits of probiotics: Are mixtures more effective than single strains? Eur. J. Nutr. 2011, 50, 1-17. [CrossRef] [PubMed]

51. Juntunen, M.; Kirjavainen, P.V.; Ouwehand, A.C.; Salminen, S.J.; Isolauri, E. Adherence of probiotic bacteria to human intestinal mucus in healthy infants and during rotavirus infection. Clin. Diagn. Lab. Immunol. 2001, 8, 293-296. [CrossRef] [PubMed]

52. Timmerman, H.M.; Koning, C.J.; Mulder, L.; Rombouts, F.M.; Beynen, A.C. Monostrain, multistrain and multispecies probiotics-A comparison of functionality and efficacy. Int. J. Food Microbiol. 2004, 96, 219-233. [CrossRef] [PubMed]

53. Zoppi, G.; Cinquetti, M.; Benini, A.; Bonamini, E.; Minelli, E.B. Modulation of the intestinal ecosystem by probiotics and lactulose in children during treatment with ceftriaxone. Curr. Ther. Res. 2001, 62, 418-435. [CrossRef]

54. Perdigon, G.; Nader de Macias, M.E.; Alvarez, S.; Oliver, G.; Pesce de Ruiz Holgado, A.A. Prevention of gastrointestinal infection using immunobiological methods with milk fermented with lactobacillus casei and lactobacillus acidophilus. J. Dairy Res. 1990, 57, 255-264. [CrossRef] [PubMed]

55. Sanders, M.E. Summary of conclusions from a consensus panel of experts on health attributes of lactic cultures: Significance to fluid milk products containing cultures. J. Dairy Sci. 1993, 76, 1819-1828. [CrossRef]

56. Ouwehand, A.C.; Isolauri, E.; Kirjavainen, P.V.; Tolkko, S.; Salminen, S.J. The mucus binding of bifidobacterium lactis bb12 is enhanced in the presence of lactobacillus gg and lact. Delbrueckii subsp. Bulgaricus. Lett. Appl. Microbiol. 2000, 30, 10-13. [CrossRef] [PubMed]

57. Klaenhammer, T.R.; Kullen, M.J. Selection and design of probiotics. Int. J. Food Microbiol. 1999, 50, 45-57. [CrossRef]

58. Lesser, L.I.; Ebbeling, C.B.; Goozner, M.; Wypij, D.; Ludwig, D.S. Relationship between funding source and conclusion among nutrition-related scientific articles. PLoS Med. 2007, 4, e5. [CrossRef] [PubMed]

59. Pedone, C.A.; Arnaud, C.C.; Postaire, E.R.; Bouley, C.F.; Reinert, P. Multicentric study of the effect of milk fermented by lactobacillus casei on the incidence of diarrhoea. Int. J. Clin. Pract. 2000, 54, 568-571. [PubMed]

(C) 2017 by the authors. Licensee MDPI, Basel, Switzerland. This article is an open access article distributed under the terms and conditions of the Creative Commons Attribution (CC BY) license (http:/ / creativecommons.org/licenses/by/4.0/). 